\title{
Sex and Age-Related Differences in Neuroticism and Allostatic Load Index in Urban Patients with General Anxiety Disorder Treated with Alprazolam
}

\author{
Carlos A. Soria ${ }^{1}$, Carolina Remedi' ${ }^{1}$, Luciana D'Alessio ${ }^{2 *}$, Emilio J. A. Roldán ${ }^{3}$ \\ ${ }^{1}$ Institute of Biosciences Henri Laborit, Córdoba, Argentina \\ ${ }^{2}$ Buenos Aires University, ENyS-CONICET, Buenos Aires, Argentina \\ ${ }^{3}$ Scientific Direction Department, Gador SA, Buenos Aires, Argentina \\ Email: ^lucianad@conicet.gov.ar, ^luladalessio@gmail.com.ar
}

How to cite this paper: Soria, C.A., Remedi, C., D'Alessio, L. and Roldán, E.J.A. (2018) Sex and Age-Related Differences in Neuroticism and Allostatic Load Index in Urban Patients with General Anxiety Disorder Treated with Alprazolam. Open Journal of Psychiatry, 8, 212-232.

https://doi.org/10.4236/ojpsych.2018.83019

Received: April 13, 2018

Accepted: June 12, 2018

Published: June 19, 2018

Copyright (c) 2018 by authors and Scientific Research Publishing Inc. This work is licensed under the Creative Commons Attribution International License (CC BY 4.0).

http://creativecommons.org/licenses/by/4.0/

\section{c) (i) Open Access}

\begin{abstract}
Introduction: Allostatic load (AL) index proposes indicators for the functioning of the main potentially stress-affected systems. Sex differences in stress response and stress-related diseases susceptibility have been described for the general population. In this observational study we describe the effects of sex and age on AL variables, in a cohort of patients with general anxiety disorders and neuroticism treated with alprazolam during 12 weeks, before and after treatment. Methods: Patients with general (DSM IV) anxiety disorders with $>6$ in Hamilton scale, AL $(>1$ Crimmins and Seeman AL modified criteria) and neuroticism $>18$ (NEO-FFI inventory), were included. All patients completed psychiatric assessment, $\mathrm{AL}$ index determination before $(-1$ week) and after 12 weeks of treatment with alprazolam $(0.25-1 \mathrm{mg} / \mathrm{t} . \mathrm{i} . \mathrm{d})$. Allostatic load parameters comprised cardiovascular, metabolic and inflammatory variables. Univariate analysis (two-way ANOVA), Student's t-test (related variables) and Pearson correlations were determined. Results: Fifty-four patients, 35 females ( $48.6 \pm 11.7$ years) and 19 males ( $44.2 \pm 12.8$ years) with general anxiety disorder were included; 28 patients with $<50$ years $(60.7 \%$ females), and 26 with $\geq 50$ years ( $69.2 \%$ females). Younger patients ( $<50$ years) (two-way ANOVA, $\mathrm{p}=0.02$ ) were significantly associated with lower AL index after treatment. However, women showed higher anxiety levels in both, before (two-way ANOVA, $\mathrm{p}=0.059$ ) and after treatment (two-way ANOVA, $p=0.005$ ), with a significantly better profile than men in many individual AL variables, particularly cardiovascular (systolic blood pressure), obesity (body mass index), and lipids (higher HDL levels). After treatment a higher reduc-
\end{abstract}


tion of fibrinogen levels was found in men (two-way ANOVA, p = 0.02). Conclusions: In this preliminary analysis we described sex and age differences in psychiatry aspects and AL indexes in patients with general anxiety disorders in the short-term treatment with alprazolam. These considerations remark the need of pondering sex and age differences during the use of drugs for protracted periods.

\section{Keywords}

Chronic Stress, Neuroticism, Alprazolam, Anxiety, Allostatic Load

\section{Introduction}

To ensure a favorable assessment of the cumulative influence of stress on health, Seeman and Crimmins first developed Allostatic Load (AL) index in 1997 and proposed indicators for the functioning of the main potentially stress-affected systems [1] [2]. AL is currently considered a marker of cumulative biological risk and describes the additive effects of multiple clinical conditions that accelerate pathophysiology, augments the vulnerability to diseases and reduces resilience [2] [3] [4] [5] [6]. AL index includes different biological markers, involving cardiovascular, metabolic and inflammatory markers [7].

Anxiety disorders, particularly general anxiety disorders (GAD) are frequently associated with chronic stress and high levels of AL [8] [9] [10] [11] [12] and also neuroticism (a clinical condition well-known as a stress vulnerability factor defined as an exaggerated response to psychosocial stressors with intense emotional reaction) [13], and mostly these factors interact increasing vulnerability to diseases, and reducing resilience [6]. Chronic stress mediated by hypothalamic-pituitary-adrenal axis (HPA) hyperactivity, has been demonstrated to induce functionally-relevant effects on hippocampus and prefrontal cortex brain neuroplasticity, that may precipitate behavioural and cognitive impairments [9] [10] [14] [15]. Treatment of patients with GAD consists in psychotherapy; particularly cognitive behavioral therapy is recommended [16]. Additionally, the use of drugs, most commonly antidepressants and/or benzodiazepines may be indicated when symptoms are severe enough to induce a significant functional impairment (National for Health and Clinical Excellence, UK guidelines) [16].

Sex differences in stress response and stress-related diseases susceptibility, have been described in the literature with controversial results [3] [17]. Regarding AL, clinical data has shown that women are less prone to cardiovascular disease and mortality than men before menopause, and metabolic and cardiovascular risk factors seem to be higher among men [18] [19] [20]. Nevertheless, women showed higher levels of inflammatory markers, but with lower rates of increase in inflammation mediators with age [20]. This situation favoring women seems to be the opposite in reference to stress-related psychiatric diseases and 
psychiatric disorders, which are more frequently observed and more severe in women than in men [3] [21]. Experimental studies demonstrated that neural remodelling pattern following chronic stress seem to be different between females and males suggesting sex differences in stress response with more intense compromise in females [3] [17]. Moreover, cognitive impairments associated to chronic stress are more intense in females [3] [17]. In addition to sex differences also age-dependent HPA axis dysregulation increase the vulnerability to stress-related disorders and contribute to cumulative AL [1] [7] [8] [12] [22] [23] [24] [25].

Despite the existence of theoretical explanations for sexual dimorphism and age effects in stress response; the empirical evidence is scarce, particularly among special populations with psychiatric disorder [19] [21] [26]. Accordingly, in this observational study we prospectively analyzed the clinical psychiatric profile and the AL index in a cohort of patients with GAD treated with alprazolam (a positive allosteric modulator of GABA A receptor) during 12 weeks, and determined the effects of sex and age on allostatic load variables before and after treatment.

\section{Methods}

\subsection{Patients}

This report is part of the preliminary results of the GEMA study protocol, which has been discussed in detail in a previous publication [27]. In this open and prospective study volunteer patients were recruited since September 2011 until May 2014, after signing the informed consent. Inclusion criteria were Hamilton Anxiety Rating $(\mathrm{HAM}-\mathrm{A})^{1}$ Scale [28] $>6$, a minimum score of 18 points in the NEO-FFI neuroticism scale [29] and at least one positive criterion for AL load index modified from Seeman and Crimmins 2003 [1] (clinical manifestations were under personalized medical treatment and were stable). All patients had a normal performance in the Mini-Mental State Examination [30] with normal vision and hearing with or without the use of aids. All patients included had an urban style of life, living in Cordoba city and surroundings. In this study, clinical and allostatic load variables were explored at baseline ( -1 week) and after treatment (12 weeks). Patients were grouped according to sex (males/females) and age ( $<50$ years $/ \geq 50$ years), being 50 years old the menopause mean age.

Exclusion criteria were: patients with depression and/or other comorbid disorders listed under axis I of the DSM-IV, (American Psychiatric Association, 1994), patients taking a psychopharmacologic, sympathomimetic, corticosteroid or any other medication that might interact with alprazolam; patients who were allergic to drugs; with confirmed or suspected pregnancy; women likely to become pregnant during the study; patients with an important clinical condition that required treatment modification and that might interfere with the study treatment or evaluation methods.

${ }^{1}$ (HAM-A) Hamilton Anxiety Rating. 


\subsection{Psychiatric Assessment}

Anxiety assessment: All patients included in this study met criteria for general anxiety disorder codified in Axis I of DSM IV (American Psychiatric Association) [31], and psychiatric assessment was determined by experimented clinical psychiatrists. In all patients the HAM-A [28] of 14 items was determined, (>6 points indicated anxiety and $\geq 15$ points indicated moderate to severe anxiety). For neuroticism assessment, the NEO-five factor inventory was applied. It measures personality factors related to neuroticism, extraversion, openness, kindness and responsibility [29].

\subsection{Allostatic Load Determination and Cut off Points}

For measuring allostatic load index, 18 clinical and biochemical parameters were determined during the morning (10 $\pm 1 \mathrm{AM})$. One point was added for each abnormal parameter up or under the cut off points according to Crimmins and Seeman criteria modified (Crimmins et al. 2003) [1]: 1-Systolic blood pressure $>140 \mathrm{mmHg}$ and/or; 2 -diastolic pressure $>90 \mathrm{mmHg}$; $3-\mathrm{BMI}^{2}$ (body mass index) $>25$; 4-waist-hip ratio (indexes of adipose tissue deposition) more than $>1$ in men and $>0.8$ in women; 5-total cholesterol $>200 \mathrm{mg} / \mathrm{dl} ; 6-\mathrm{LDL}$ (low density lipoprotein) $>120 \mathrm{mg} / \mathrm{dl}$; 7-HDL (high density lipoprotein) $>37$ $\mathrm{mg} / \mathrm{dl} ; 8$-total cholesterol/HDL ratio $>3.5 \mathrm{mg} / \mathrm{dl}$; 9-triglycerides $>200 \mathrm{mg} / \mathrm{dl}$; 10 -creatinine $>1.2 \mathrm{mg} / \mathrm{dl} ; 11$-albumin $<3.5 \mathrm{~g} / \mathrm{dl} ; 12$-C-reactive protein (CRP) $>7.1 \mathrm{mg} / \mathrm{L}$; 13-fibrinogen $>400 \mathrm{mg} / \mathrm{dl}$; 14 -glycated hemoglobin $>6 \mathrm{mg} / \mathrm{dl}$; 15-salivary cortisol $>32 \mathrm{nM}$; 16-salivary methoxy-hydroxy-phenylglycol (MHPG) (noradrenaline metabolite) $>2750 \mathrm{nM}$; 17-serum dehydro-epi-androsterone (DHEA) $<80 \mathrm{ng} / \mathrm{ml}$ in men and $<35 \mathrm{ng} / \mathrm{ml}$ in women; 18-serum noradrenaline levels $>100 \mathrm{pg} / \mathrm{ml}$. The study participants were evaluated prior to treatment (week-1) and after treatment initiation (week 12).

\subsection{General Conditions and Safety}

Additional assessments included respiratory function and laboratory tests. All patients have been warned about alprazolam's depressant effects and the possible risks in activities that required alert. Alcohol consumption was not recommended. Patients were also monitored to detect risk of suicide and/or overdose. All adverse events were monitored according to good clinical practice standards of the local regulatory authority; National Administration of Drugs, Foods and Medical Devices (ANMAT) and the Ministry of Health, Province of Córdoba.

\subsection{Treatment Instauration}

Patients admitted into the study were given alprazolam tablets (Alplax ${ }^{\oplus}$, Gador SA, Buenos Aires; batch number 03730), in a flexible dosing regimen within 0.25 to $1 \mathrm{mg}$ t.i.d. (three times a day), which enabled the analysis of the dose-response relationship for the variables under investigation. Individual doses were deter${ }^{2}$ (BMI) Body mass index. 
mined according to clinical criteria by the researcher responsible for each patient, and the lowest effective dose was administered in each case. Patient compliance was assessed by monitoring salivary alprazolam levels and found to be satisfactory throughout the study in all cases. The alprazolam's dosage schedule was maintained for 12 weeks. Subsequently, the investigator decided to continue or discontinue treatment. Laboratory tests were blinded to the researcher. Statistics and graphs were obtained using a computational program for windows.

\subsection{Statistics}

Dispersion measures of quantitative variables were determined. Statistical significance was considered at $\mathrm{p}<0.05$ ( 2 -sided; $1-\beta$ power $\geq 0.80$ ). The sample size was calculated in $(n=29-55)$ cases, with the aim to detect changes in variables higher than $25 \%$ with a $1-\beta$ power of $0-80$ [27].

Spearman (non-parametric) and/or Pearson (parametric) tests were used to determine the correlation coefficient: $r>0.80$ and $p<0.05$ (2-sided) was considered as strong correlation and $0.50<\mathrm{r}<0.80$ and $\mathrm{p}<0.05$ (2-sided), was considered a moderate correlation [32]. For non-continuous variables Chi-square test and/or Fisher's test was determined. To analyze sex and age factors effects on clinical and allostatic load parameters, a general univariant lineal model (two-way ANOVA) was applied for each allostatic load variable measured before and after treatment. Student's t-test related variables were used to compare measures before and after treatment. Two-way ANOVA test was used to determine the effects of sex and age on the arithmetic differences determined after treatment for each $\mathrm{AL}$ parameter. Interactions between factors were also analyzed, and the effect size was calculated using the partial eta squared $\left(\mathrm{hp}^{2}\right)$. Pearson/Spearman correlations were determined for continuous variables.

\subsection{Approval}

The protocol was evaluated and approved in accordance with the code of ethics of the World Medical Association (Declaration of Helsinki) and Clinical Practice guidelines in Argentina, by the Independent Ethics Committee of the Foundation for Pharmacological Studies and Drugs, Buenos Aires, and then submitted to the national regulatory authority (ANMAT, Disposition \#61409-8) and to the regulatory authority, under the Ministry of Health's responsibility, Province of Córdoba (Dossier \#1296). The trial is also registered at WHO (Word Health Organization $)^{3}$, trial registration data set. Results were reported to the ANMAT, Argentine regulatory agency in accordance with regulations in force. All patients signed the informed consent form.

\section{Results}

In this observational study, 54 patients, 35 females ( $48.6 \pm 11.7$ years) and 19 males (44.2 \pm 12.8 years) with general anxiety disorder were included. Patients ${ }^{3}(\mathrm{WHO})$ Word Health Organization. 
were also grouped by age resulting in 28 patients with $<50$ years ( $60.7 \%$ females), and $26 \geq 50$ years (69.2\% females). From this group 50 patients, 31 females and 19 males completed the treatment period of 12 weeks. No serious adverse events were reported.

Table 1 summarizes the clinical psychiatric variables and the total AL index. Women showed a different psychiatric profile with higher anxiety levels in the HAM-A at both, before and after treatment, but at the same time with a better profile in neuroticism scale showing higher scores in NEOFFI kindness before and after treatment. Additionally, HAM-A index improved in all groups after 12 weeks of treatment (Student $t$ test, $p<0.05$ ). Younger patients $(<50$ years) but non-sex related factor were associated with significantly lower AL index after treatment.

Table 2 summarizes the individual AL parameters. Women showed a significantly better profile in many AL indexes, particularly in the cardiovascular ones, with lower systolic blood pressure, higher HDL levels and lower total cholesterol (Tch) and Tch/HDL ratio before and after treatment compared to men, without significant interactions with age-related factor $(\mathrm{p}>0.05)$. Furthermore, women showed lower body mass index, lower waist-hip ratio, and lower creatinine levels before and after treatment and lower levels of triglycerides before treatment compared to men, without significant interactions with age-related factor.

In a further analysis (Table 3), the univariate analysis for age and sex was calculated for the arithmetic differences observed after treatment in all AL parameters measured, and the student's t-test for related variables was calculated for the mean of each variable measured. It can be noticed that a higher reduction of fibrinogen levels and Tch/HDL ratio was detected in men compared to women, without significant interactions with age-related factor, along with differences among pharmacological responses to alprazolam.

Finally, Pearson correlations between AL and clinical variables were determined, and some findings were assessed regarding inflammatory markers. A low to moderate positive correlation between BMI and CRP was found in women before $(\mathrm{r}=0.54, \mathrm{p}=0.001)$, and after treatment $(\mathrm{r}=0.59, \mathrm{p}=0.001)$, while in men it was slightly lower $(\mathrm{r}=0.48, \mathrm{p}=0.039)$ and $(\mathrm{r}=0.45, \mathrm{p}=0.051)$ before and after treatment, respectively. Other correlations were lower and negative as the fibrinogen and Tch, $(\mathrm{r}=-0.46, \mathrm{p}=0.047)$ in men and $(\mathrm{r}=-0.48, \mathrm{p}=0.004)$ in women, observed before treatment, but not following treatment. In addition, only in women CRP negatively correlated with HDL $(r=-0.36, p=0.045)$, and fibrinogen negatively correlated with $\operatorname{HDL}(-0.40, \mathrm{p}=0.027)$. Additionally after treatment, a low to moderate negative correlation between CRP and plasmatic cortisol $(r=-0.44, p=0.015)$ was found in women, but not in men $(r=-0.16, p$ $=0.504)$.

\section{Discussion}

These preliminary results regarding AL index distribution by sex and age in 
Table 1. Comparison of clinical psychiatric assessments (Hamilton-Anxiety and NEOFFI scales) and total allostatic load index, in a sample of patients with anxiety disorders, neuroticism and allostatic load, clustered by sex and age groups ( $\geq 50$ years; $<50$ years), previous (basal) and after 12 weeks of alprazolam low dose treatment.

\begin{tabular}{|c|c|c|c|c|c|c|c|}
\hline $\begin{array}{c}\text { Clinical } \\
\text { Assessments } \\
\text { (scores) }\end{array}$ & $\begin{array}{l}\text { Sex } \\
\text { Age }\end{array}$ & $\mathbf{n}$ & $\chi$ & SD & $\begin{array}{c}\text { Two-way } \\
\text { ANOVA } \\
\text { F (df) } \\
=p \text { value }\end{array}$ & $\begin{array}{c}\text { Partial eta } \\
\text { squared } \\
\left(\mathrm{hp}^{2}\right)\end{array}$ & $\begin{array}{c}t \text {-test } \\
\text { (basal vs. } \\
\text { w12 ) } \\
\text { p value }\end{array}$ \\
\hline \multicolumn{8}{|c|}{ Hamilton Anxiety Scale (HAM-A) } \\
\hline \multirow{4}{*}{ Basal } & Females & 35 & 31.7 & 10.2 & $3.7(1.50)$ & \multirow{2}{*}{0.069} & \\
\hline & Males & 19 & 28.2 & 6.4 & $=0.05$ & & \\
\hline & $\geq 50$ ys & 26 & 31.4 & 8.1 & $0.01(1.50)$ & \multirow{2}{*}{0.001} & \\
\hline & $<50$ ys & 28 & 30.9 & 8.3 & $=0.97$ & & \\
\hline \multirow{4}{*}{ w12 } & Females & 31 & 14.4 & 5.7 & $8.82(1.46)$ & \multirow{2}{*}{0.161} & $0.001^{*}$ \\
\hline & Males & 19 & 9.4 & 5.8 & $=0.005^{\star}$ & & $0.001^{*}$ \\
\hline & $\geq 50$ ys & 24 & 13.1 & 6.4 & $0.03(1.46)$ & \multirow{2}{*}{0.000} & $0.001^{*}$ \\
\hline & $<50$ ys & 26 & 11.9 & 6.1 & $=0.90$ & & $0.001^{*}$ \\
\hline \multicolumn{8}{|c|}{ NEOFFI Neuroticism } \\
\hline \multirow{4}{*}{ Basal } & Females & 35 & 31.6 & 6.7 & $0.09(1.50)$ & \multirow{2}{*}{0.002} & \\
\hline & Males & 19 & 30.9 & 6.7 & $=0.75$ & & \\
\hline & $\geq 50$ ys & 26 & 30.5 & 6.02 & $0.34(1.50)$ & \multirow{2}{*}{0.007} & \\
\hline & $<50$ ys & 28 & 32.1 & 7.2 & $=0.55$ & & \\
\hline \multirow{4}{*}{ w12 } & Females & 31 & 29.2 & 7.3 & $0.44(1.46)$ & \multirow{2}{*}{0.010} & $0.021^{*}$ \\
\hline & Males & 19 & 28.3 & 7.2 & $=0.50$ & & 0.085 \\
\hline & $\geq 50$ ys & 24 & 28.0 & 7.0 & $1.82(1.46)$ & \multirow{2}{*}{0.038} & $0.024^{*}$ \\
\hline & $<50$ ys & 26 & 29.7 & 7.4 & $=0.18$ & & 0.055 \\
\hline \multicolumn{8}{|c|}{ NEOFFI Extroversion } \\
\hline \multirow{5}{*}{ Basal } & Females & 35 & 25.2 & 8.9 & $0.59(1.50)$ & \multirow{2}{*}{0.012} & \\
\hline & Males & 19 & 23.5 & 6.8 & $=0.44$ & & \\
\hline & & & & & & \multirow{3}{*}{0.037} & \\
\hline & $\geq 50$ ys & 26 & 22.6 & 6.6 & $1.93(1.50)$ & & \\
\hline & $<50$ ys & 28 & 26.4 & 9.1 & $=0.17$ & & \\
\hline \multirow{4}{*}{ w12 } & Females & 31 & 26.8 & 9.0 & $1.26(1.46)$ & \multirow{2}{*}{0.027} & 0.142 \\
\hline & Males & 19 & 24.3 & 7.5 & $=0.27$ & & 0.577 \\
\hline & $\geq 50$ ys & 24 & 23.5 & 8.5 & $2.49(1.46)$ & \multirow{3}{*}{0.051} & 0.448 \\
\hline & $<50 \mathrm{ys}$ & 26 & 28.0 & 7.9 & $=0.12$ & & 0.186 \\
\hline \multicolumn{7}{|c|}{ NEOFFI Openness } & \\
\hline \multirow{5}{*}{ Basal } & Females & 35 & 25.7 & 7.8 & $0.04(1.50)$ & \multirow{2}{*}{0.001} & \\
\hline & Males & 19 & 26.2 & 5.5 & $=0.84$ & & \\
\hline & & 26 & 24.5 & & & \multirow{3}{*}{0.019} & \\
\hline & $250 \mathrm{ys}$ & & & & $0.96(1.50)$ & & \\
\hline & $<50$ ys & 28 & 27.1 & 7.7 & $=0.33$ & & \\
\hline
\end{tabular}




\section{Continued}

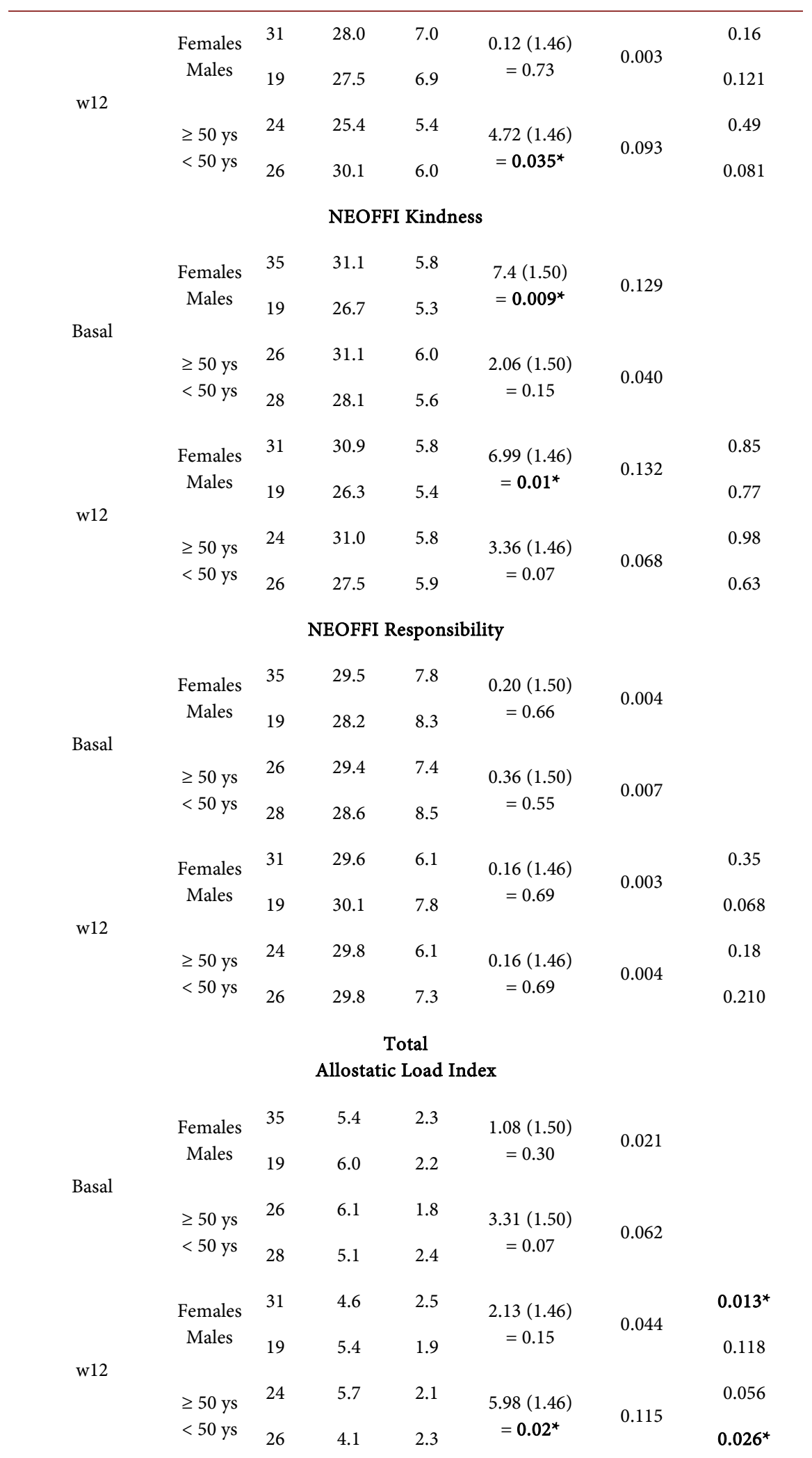

$\mathrm{n}$ : number of subjects per group; $\chi$ : average value; SD: standard deviation from mean; df: degrees of freedom, basal: week 0 of treatment, w12: week 12 of treatment, ys: years, vs: versus, ${ }^{*} \mathrm{p}<0.05$ (significant outcome). 
Table 2. Comparison of the individual components of the allostatic load index assessments, in a sample of patients with anxiety disorders, neuroticism and allostatic load, clustered by sex and age groups ( $\geq 50$ years; $<50$ years), previous (basal) and after 12 weeks of alprazolam low dose treatment. See the cut off points for each variable in the text.

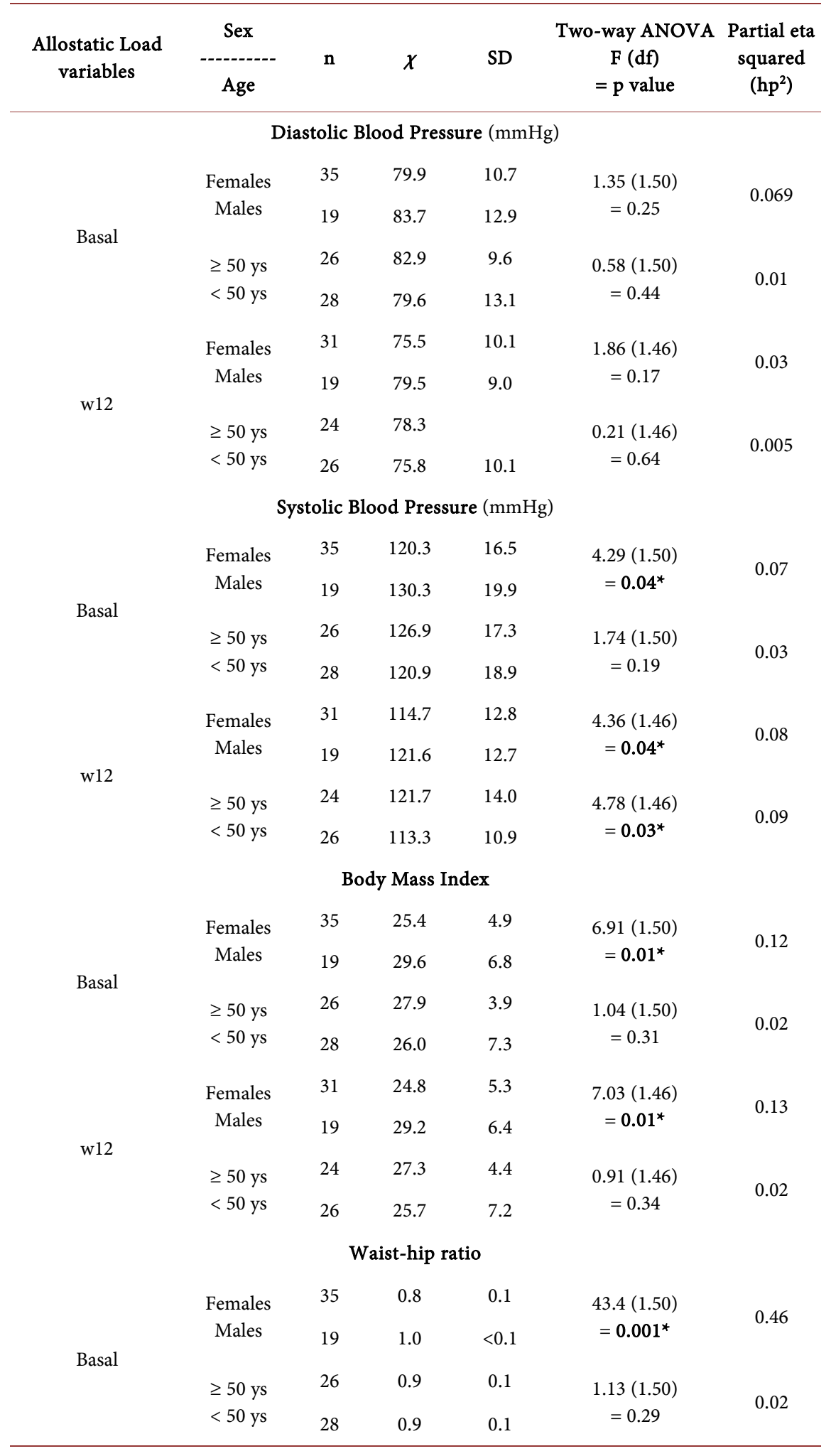




\section{Continued}

\begin{tabular}{|c|c|c|c|c|c|c|}
\hline & Females & 31 & 0.8 & 0.1 & $41.95(1.46)$ & \multirow{2}{*}{0.47} \\
\hline \multirow{4}{*}{ w12 } & Males & 19 & 1.0 & 0.1 & $=0.001^{*}$ & \\
\hline & & & & & & \\
\hline & $\geq 50$ ys & 24 & 0.9 & 0.1 & $2.97(1.46)$ & \multirow{2}{*}{0.06} \\
\hline & $<50$ ys & 26 & 0.9 & 0.1 & $=0.09$ & \\
\hline \multicolumn{7}{|c|}{ Total Cholesterol (mg/dl) } \\
\hline \multirow{5}{*}{ Basal } & Females & 35 & 225.3 & 41.4 & $3.18(1.50)$ & \multirow{2}{*}{0.06} \\
\hline & Males & 19 & 204.3 & 41.0 & $=0.08$ & \\
\hline & & & & & & \multirow{3}{*}{0.001} \\
\hline & $\geq 50$ ys & 26 & 220.9 & 47.5 & $0.01(1.50)$ & \\
\hline & $<50$ ys & 28 & 215.0 & 37.0 & $=0.90$ & \\
\hline \multirow{5}{*}{ w12 } & Females & 31 & 215.2 & 44.8 & $2.33(1.46)$ & \multirow{2}{*}{0.0} \\
\hline & Males & 19 & 193.6 & 46.2 & $=0.13$ & \\
\hline & & & & & & \multirow{3}{*}{0.02} \\
\hline & $\geq 50$ ys & 24 & 215.0 & 51.7 & $0.94(1.46)$ & \\
\hline & $<50$ ys & 26 & 199.5 & 39.7 & $=0.33$ & \\
\hline \multicolumn{7}{|c|}{ Cholesterol LDL (mg/dl) } \\
\hline \multirow{5}{*}{ Basal } & Females & 35 & 136.8 & 31.7 & $1.39(1.50)$ & \multirow{2}{*}{0.02} \\
\hline & Males & 19 & 126.2 & 40.0 & $=0.24$ & \\
\hline & & & & & & \multirow{3}{*}{0.008} \\
\hline & $\geq 50$ ys & 26 & 131.9 & 35.4 & $0.39(1.50)$ & \\
\hline & $<50$ ys & 28 & 134.2 & 34.9 & $=0.53$ & \\
\hline \multirow{5}{*}{ w12 } & Females & 31 & 126.4 & 33.2 & $0.02(1.46)$ & \multirow{2}{*}{0.001} \\
\hline & Males & 19 & 124.5 & 39.2 & $=0.86$ & \\
\hline & & & 127 & 300 & & \multirow{3}{*}{0.001} \\
\hline & $\geq 50$ ys & 24 & 127.1 & 39.2 & $0.03(1.46)$ & \\
\hline & $<50$ ys & 26 & 124.4 & 31.8 & $=0.86$ & \\
\hline \multicolumn{7}{|c|}{ Cholesterol HDL (mg/dl) } \\
\hline \multirow{4}{*}{ Basal } & Females & 35 & 61.4 & 16.7 & $17.57(1.50)$ & \multirow{2}{*}{0.26} \\
\hline & Males & 19 & 43.0 & 10.3 & $=0.001^{*}$ & \\
\hline & & 26 & 57.3 & 16.4 & & \multirow[b]{2}{*}{0.01} \\
\hline & $<50$ ys & 28 & 52.7 & 17.8 & $=0.46$ & \\
\hline \multirow{5}{*}{ w12 } & Females & 31 & 61.1 & 15.9 & $11.60(1.46)$ & \multirow{2}{*}{0.20} \\
\hline & Males & 19 & 45.5 & 13.0 & $=0.001^{*}$ & \\
\hline & & & & & & \multirow{3}{*}{0.01} \\
\hline & $\geq 50$ ys & 24 & 57.0 & 16.6 & $0.63(1.46)$ & \\
\hline & $<50$ ys & 26 & 53.5 & 16.7 & $=0.43$ & \\
\hline & & & o $\mathrm{TCh} /$ & & & \\
\hline & Females & 35 & 3.9 & 1.3 & $6.74(1.50)$ & 011 \\
\hline & Males & 19 & 5.0 & 1.3 & $=0.01^{*}$ & 0.11 \\
\hline Basal & & & & & & \\
\hline & $\geq 50$ ys & 26 & 4.1 & 1.1 & $0.91(1.50)$ & 01 \\
\hline & $<50$ ys & 28 & 4.5 & 1.6 & $=0.34$ & 0.01 \\
\hline
\end{tabular}




\section{Continued}

\begin{tabular}{|c|c|c|c|c|c|c|}
\hline \multirow{5}{*}{ w12 } & Females & 31 & 3.8 & 1.2 & $3.72(1.46)$ & \multirow{2}{*}{0.07} \\
\hline & Males & 19 & 4.4 & 1.1 & $=0.06$ & \\
\hline & & & & & & \\
\hline & $\geq 50$ ys & 24 & 4.0 & 1.2 & $0.001(1.46)$ & \multirow{2}{*}{0.001} \\
\hline & $<50 \mathrm{ys}$ & 26 & 4.0 & 1.2 & $=0.98$ & \\
\hline \multicolumn{7}{|c|}{ Triglycerides (mg/dl) } \\
\hline \multirow{5}{*}{ Basal } & Females & 35 & 142.0 & 87.1 & $4.53(1.50)$ & \multirow{2}{*}{0.08} \\
\hline & Males & 19 & 195.4 & 98.1 & $=0.03^{*}$ & \\
\hline & & & & & & \multirow{3}{*}{0.01} \\
\hline & $\geq 50$ ys & 26 & 168.4 & 98.4 & $0.75(1.50)$ & \\
\hline & $<50$ ys & 28 & 153.7 & 90.4 & $=0.38$ & \\
\hline \multirow{5}{*}{ w12 } & Females & 31 & 137.8 & 79.96 & $1.74(1.46)$ & \multirow{2}{*}{0.036} \\
\hline & Males & 19 & 162.3 & 66.12 & $=0.19$ & \\
\hline & & & & & & \multirow{3}{*}{0.09} \\
\hline & $\geq 50$ ys & 24 & 172.3 & 86.12 & $4.85(1.46)$ & \\
\hline & $<50$ ys & 26 & 123.9 & 55.73 & $=0.03^{*}$ & \\
\hline \multicolumn{7}{|c|}{ Creatinine $(\mathrm{mg} / \mathrm{dl})$} \\
\hline \multirow{5}{*}{ Basal } & Females & 35 & 0.7 & 0.1 & $56.09(1.50)$ & \multirow{2}{*}{0.52} \\
\hline & Males & 19 & 1.0 & 0.2 & $=0.001^{\star}$ & \\
\hline & & & & & & \multirow{3}{*}{0.03} \\
\hline & $\geq 50$ ys & 26 & 0.8 & 0.2 & $1.79(1.50)$ & \\
\hline & $<50$ ys & 28 & 0.8 & 0.2 & $=0.18$ & \\
\hline \multirow{5}{*}{ w12 } & Females & 31 & 0.7 & 0.1 & $39.92(1.46)$ & \multirow{2}{*}{0.46} \\
\hline & Males & 19 & 0.9 & 0.1 & $=0.001^{*}$ & \\
\hline & & & & & & \multirow{3}{*}{0.001} \\
\hline & $\geq 50$ ys & 24 & 0.8 & 0.8 & $0.005(1.46)$ & \\
\hline & $<50$ ys & 26 & 0.8 & 0.2 & $=0.94$ & \\
\hline \multicolumn{7}{|c|}{ Albumin (g/dl) } \\
\hline \multirow{5}{*}{ Basal } & Females & 35 & 4.6 & 0.4 & $0.71(1.50)$ & \multirow{2}{*}{0.01} \\
\hline & Males & 19 & 4.5 & 0.4 & $=0.40$ & \\
\hline & & 26 & 45 & 04 & & \multirow{3}{*}{0.03} \\
\hline & $\geq 50 \mathrm{ys}$ & 26 & 4.5 & 0.4 & $1.70(1.50)$ & \\
\hline & $<50$ ys & 28 & 4.60 & 0.3 & $=0.19$ & \\
\hline \multirow{5}{*}{ w12 } & Females & 31 & 4.54 & 0.46 & $0.40(1.46)$ & \multirow{2}{*}{0.009} \\
\hline & Males & 19 & 4.5 & 0.5 & $=0.53$ & \\
\hline & & & & & & \multirow{3}{*}{0.008} \\
\hline & $\geq 50 \mathrm{ys}$ & 24 & 4.5 & 0.5 & $0.35(1.46)$ & \\
\hline & $<50$ ys & 26 & 4.5 & 0.4 & $=0.55$ & \\
\hline & & $\mathrm{Re}$ & e Prot & $(\mathrm{mg} / \mathrm{L})$ & & \\
\hline & Females & 35 & 2.2 & 3.7 & $0.15(1.50)$ & 0003 \\
\hline & Males & 19 & 1.8 & 2.7 & $=0.70$ & 0.003 \\
\hline Basal & & & & & & \\
\hline & $\geq 50$ ys & 26 & 1.9 & 2.7 & $0.08(1.50)$ & ? \\
\hline & $<50$ ys & 28 & 2.3 & 3.9 & $=0.78$ & 0.002 \\
\hline
\end{tabular}




\section{Continued}

\begin{tabular}{|c|c|c|c|c|c|c|}
\hline & Females & 31 & 1.6 & 2.3 & $0.13(1.46)$ & \multirow{2}{*}{0.003} \\
\hline \multirow{4}{*}{ w12 } & Males & 19 & 1.5 & 1.4 & $=0.71$ & \\
\hline & & & & & & \\
\hline & $\geq 50$ ys & 24 & 1.5 & 1.9 & $0.20(1.46)$ & \multirow{2}{*}{0.004} \\
\hline & $<50$ ys & 26 & 1.6 & 2.1 & $=0.65$ & \\
\hline \multicolumn{7}{|c|}{ Fibrinogen $(\mathrm{mg} / \mathrm{dl})$} \\
\hline \multirow{5}{*}{ Basal } & Females & 35 & 394.4 & 139.8 & $0.29(1.50)$ & \multirow{2}{*}{0.006} \\
\hline & Males & 19 & 414.4 & 107.2 & $=0.59$ & \\
\hline & & & & & & \multirow{3}{*}{0.01} \\
\hline & $\geq 50$ ys & 26 & 420.1 & 126.5 & $0.51(1.50)$ & \\
\hline & $<50$ ys & 28 & 384.1 & 130.4 & $=0.47$ & \\
\hline \multirow{5}{*}{ w12 } & Females & 31 & 424.3 & 152.2 & $0.70(1.46)$ & \multirow{2}{*}{0.01} \\
\hline & Males & 19 & 383.5 & 93.6 & $=0.40$ & \\
\hline & & & & & & \multirow{3}{*}{0.004} \\
\hline & $\geq 50$ ys & 24 & 418.8 & 133.0 & $0.19(1.46)$ & \\
\hline & $<50$ ys & 26 & 399.6 & 135.7 & $=0.66$ & \\
\hline \multicolumn{7}{|c|}{ Glycated Hemoglobin (mg/dl) } \\
\hline \multirow{5}{*}{ Basal } & Females & 35 & 6.0 & 0.8 & $0.70(1.50)$ & \multirow{2}{*}{0.01} \\
\hline & Males & 19 & 5.8 & 0.5 & $=0.40$ & \\
\hline & & & & & & \multirow{3}{*}{0.004} \\
\hline & $\geq 50$ ys & 26 & 6.0 & 0.7 & $0.19(1.50)$ & \\
\hline & $<50$ ys & 28 & 5.8 & 0.7 & $=0.66$ & \\
\hline \multirow{5}{*}{ w12 } & Females & 31 & 5.7 & 0.6 & $0.46(1.46)$ & \multirow{2}{*}{0.01} \\
\hline & Males & 19 & 5.8 & 0.6 & $=0.50$ & \\
\hline & & & & & & \multirow{3}{*}{0.07} \\
\hline & $\geq 50$ ys & 24 & 5.9 & 0.7 & $3.69(1.46)$ & \\
\hline & $<50$ ys & 26 & 5.6 & 0.4 & $=0.06$ & \\
\hline \multicolumn{7}{|c|}{ Salivary Cortisol (nM) } \\
\hline \multirow{5}{*}{ Basal } & Females & 35 & 13.0 & 9.9 & $0.27(1.50)$ & \multirow{2}{*}{0.005} \\
\hline & Males & 19 & 13.8 & 5.3 & $=0.60$ & \\
\hline & & 26 & 120 & 73 & & \multirow{3}{*}{0.001} \\
\hline & $\geq 50$ ys & 20 & 12.0 & 1.0 & $0.04(1.50)$ & \\
\hline & $<50$ ys & 28 & 14.5 & 9.4 & $=0.85$ & \\
\hline \multirow{5}{*}{ w12 } & Females & 31 & 9.9 & 7.5 & $0.09(1.46)$ & \multirow{2}{*}{0.002} \\
\hline & Males & 19 & 10.4 & 8.7 & $=0.76$ & \\
\hline & & & & & & \multirow{3}{*}{0.001} \\
\hline & $\geq 50$ ys & 24 & 10.0 & 8.8 & $0.05(1.46)$ & \\
\hline & $<50$ ys & 26 & 10.2 & 7.0 & $=0.83$ & \\
\hline & & Sal & y MHP & $(\mathrm{nM})$ & & \\
\hline & Females & 35 & 1655.8 & 1516.3 & $0.56(1.50)$ & 0.1 \\
\hline & Males & 19 & 2126.6 & 2638.7 & $=0.45$ & 0.01 \\
\hline Basal & & & & & & \\
\hline & $\geq 50 \mathrm{ys}$ & 26 & 1700.3 & 1515.9 & $0.19(1.50)$ & \\
\hline & $<50$ ys & 28 & 1933.9 & 2341.6 & $=0.66$ & 0.004 \\
\hline
\end{tabular}




\section{Continued}

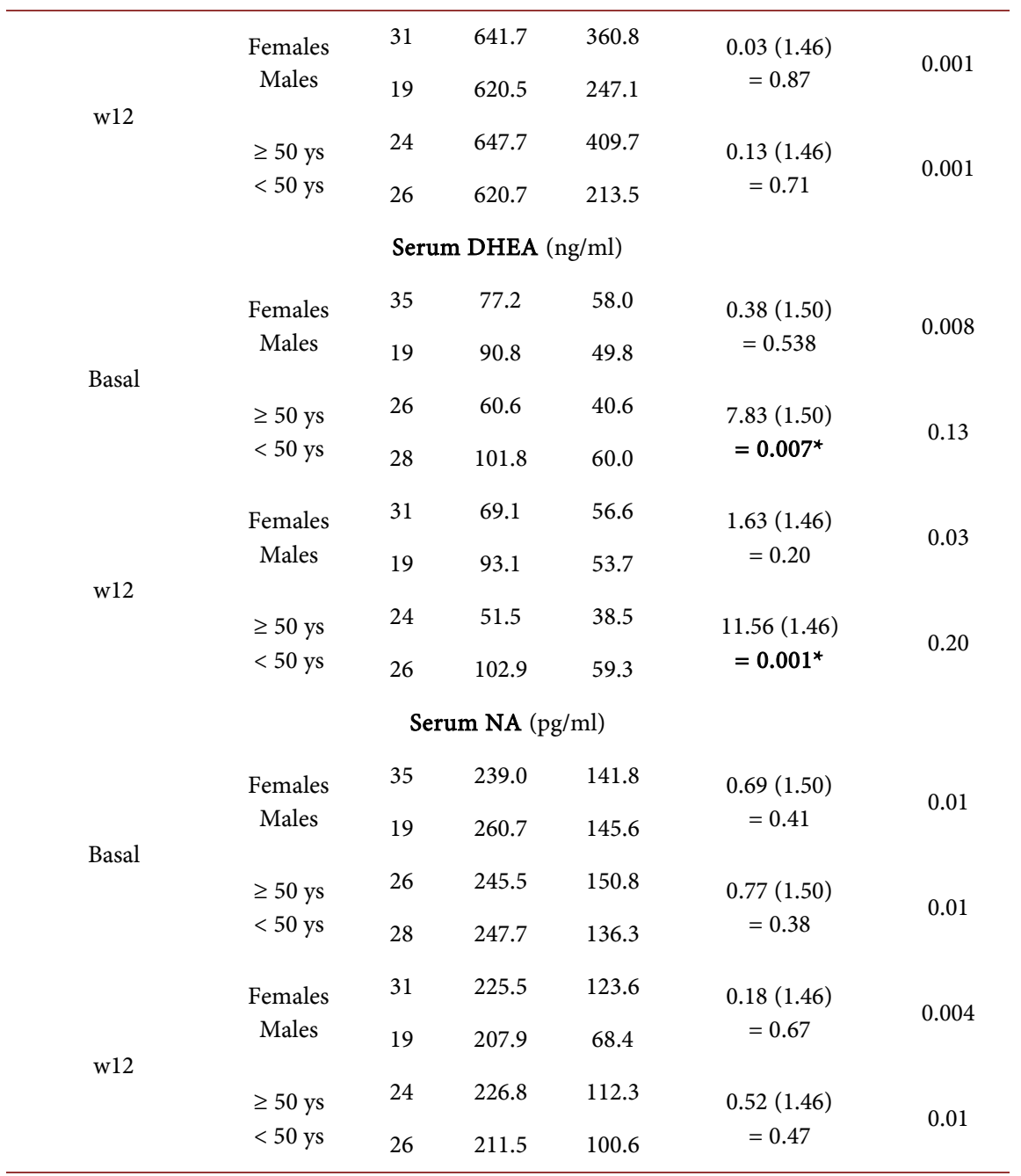

$\mathrm{n}$ : number of subjects per group; $\chi$ : average value; SD: standard deviation from mean; df: degrees of freedom, basal: week 0 of treatment, w12: week 12 of treatment, ys: years, MHPG: Methoxy-hydroxy-phenylglycol, DHEA: dehidro-epiandrosterone, NA: noradrenaline, ${ }^{*} \mathrm{p}<0.05$ (significant outcome).

Table 3. Differences in the total allostatic load index and in its individual variables in a sample of patients with anxiety disorders, neuroticism and allostatic load, clustered by sex and age groups ( $\geq 50$ years; $<50$ years), previous (basal) and after 12 weeks of alprazolam low dose treatment. The figures ( $\chi$ and SD) show the arithmetic difference between the values before and after treatment. Two-way ANOVA test the significance between groups (by sex or age), and test-t test the difference between before and after for each variable.

\begin{tabular}{ccccccc}
\hline $\begin{array}{c}\text { Arithmetic } \\
\text { difference } \\
\text { after treatment }\end{array}$ & Sex & Age & n & $\chi$ & SD & $\begin{array}{c}\text { Two-way ANOVA Partial eta } \\
\text { F (df) } \\
\text { squared }\end{array}$ \\
\hline & $\begin{array}{c}\text { Females } \\
\left.\text { (hp }{ }^{2}\right)\end{array}$ & 31 & -0.74 & 1.56 & $\begin{array}{c}0.13(1.46) \\
=0.72\end{array}$ & 0.069 \\
$\begin{array}{c}\text { Total Allostatic } \\
\text { Load Index } \\
\text { (score) }\end{array}$ & Males & 19 & -0.57 & 1.53 & & \\
& $\geq 50$ ys & 24 & -0.54 & 1.31 & $\begin{array}{c}0.11(1.46) \\
=0.74\end{array}$ & 0.002 \\
\hline
\end{tabular}




\section{Continued}

\begin{tabular}{|c|c|c|c|c|c|c|}
\hline \multirow{4}{*}{$\begin{array}{c}\text { Diastolic Blood } \\
\text { Pressure } \\
\text { (mmHg) }\end{array}$} & Females & 31 & -4.26 & 9.05 & $0.01(1.46)$ & \multirow{2}{*}{0.001} \\
\hline & Males & 19 & -4.21 & 16.18 & \multirow[t]{2}{*}{$=0.93$} & \\
\hline & \multirow{2}{*}{$\begin{array}{l}\geq 50 \mathrm{ys} \\
<50 \mathrm{ys}\end{array}$} & 24 & -5.00 & 10.89 & & \multirow[b]{2}{*}{0.008} \\
\hline & & 26 & -3.57 & 12.97 & $\begin{array}{c}0.37(1.46) \\
\mathrm{p}=0.54\end{array}$ & \\
\hline \multirow{4}{*}{$\begin{array}{c}\text { Systolic Blood } \\
\text { Pressure } \\
\text { (mmHg) }\end{array}$} & $\begin{array}{c}\text { Females } \\
\text { Males }\end{array}$ & 31 & -8.68 & 16.98 & \multirow{2}{*}{$\begin{array}{c}0.46(1.46) \\
=0.42\end{array}$} & \multirow{2}{*}{0.01} \\
\hline & Males & 19 & -5.32 & 14.37 & & \\
\hline & $\geq 50 \mathrm{ys}$ & 24 & -6.66 & 15.01 & \multirow{2}{*}{$\begin{array}{c}0.07(1.46) \\
=0.78\end{array}$} & \multirow{2}{*}{0.002} \\
\hline & $<50 \mathrm{ys}$ & 26 & -6.53 & 15.92 & & \\
\hline \multirow{5}{*}{ Body Mass Index } & \multirow{2}{*}{$\begin{array}{c}\text { Females } \\
\text { Males }\end{array}$} & 31 & -0.41 & 1.65 & \multirow{3}{*}{$\begin{array}{c}0.00(1.46) \\
=0.99\end{array}$} & \multirow{2}{*}{0.001} \\
\hline & & 19 & -0.43 & 1.19 & & \\
\hline & \multirow{3}{*}{$\begin{array}{l}\geq 50 \text { ys } \\
<50 \text { ys }\end{array}$} & & & & & \\
\hline & & 24 & -0.58 & 1.88 & \multirow{2}{*}{$\begin{array}{c}0.18(1.46) \\
=0.67\end{array}$} & \multirow{2}{*}{0.004} \\
\hline & & 26 & -0.27 & 1.00 & & \\
\hline \multirow{5}{*}{ Waist-hip ratio } & \multirow{2}{*}{$\begin{array}{c}\text { Females } \\
\text { Males }\end{array}$} & 31 & 0.002 & 0.03 & $0.15(1.46)$ & \multirow{2}{*}{0.003} \\
\hline & & 19 & 0.005 & 0.04 & $=0.69$ & \\
\hline & & & & & & \\
\hline & $\geq 50 \mathrm{ys}$ & 24 & 0.009 & 0.03 & $1.20(1.46)$ & 002 \\
\hline & $<50$ ys & 26 & -0.002 & 0.04 & $=0.27$ & 0.02 \\
\hline & Females & 31 & -12.48 & 35.70 & $0.09(1.46)$ & 0.002 \\
\hline Total Cholesterol & Males & 19 & -10.68 & 37.44 & $=0.76$ & 0.002 \\
\hline (mg/dl) & $\geq 50 \mathrm{ys}$ & 24 & -6.70 & 36.49 & $1.14(1.46)$ & \\
\hline & $<50$ ys & 26 & -16.50 & 35.59 & $=0.29$ & 0.02 \\
\hline & Females & 31 & -11.97 & 30.05 & $1.97(1.46)$ & 004 \\
\hline $\begin{array}{c}\text { Cholesterol } \\
\text { LDL }\end{array}$ & Males & 19 & -1.63 & 23.34 & $=0.16$ & 0.04 \\
\hline$(\mathrm{mg} / \mathrm{dl})$ & $\geq 50$ ys & 24 & -8.04 & 27.90 & $0.61(1.46)$ & 001 \\
\hline & $<50$ ys & 26 & -9.57 & 28.28 & $=0.43$ & 0.01 \\
\hline & Females & 31 & -1.61 & 12.55 & 1.85 & 003 \\
\hline Cholesterol & Males & 19 & 2.52 & 8.60 & $=0.17$ & 0.00 \\
\hline$(\mathrm{mg} / \mathrm{dl})$ & $\geq 50$ ys & 24 & -0.54 & 10.01 & $0.03(1.46$ & 0.001 \\
\hline & $<50$ ys & 26 & 0.42 & 12.56 & $=0.85$ & \\
\hline & Females & 31 & -0.14 & 0.64 & $3.54(1.46)$ & 0.07 \\
\hline & Males & 19 & -0.53 & 0.73 & $=0.06$ & 0.07 \\
\hline Katio ICA/HDL & & 24 & -0.06 & 0.56 & & \\
\hline & $\geq 50$ ys & 24 & -0.00 & 0.56 & $3.41(1.46)$ & \\
\hline & $<50$ ys & 26 & -0.49 & 0.75 & $=0.07$ & 0.06 \\
\hline & Females & 31 & -0.51 & 74.00 & & \\
\hline Triglycerides & Males & 19 & -33.10 & 65.99 & $\begin{array}{c}2.52(1.46) \\
=0.12\end{array}$ & 0.05 \\
\hline$(\mathrm{mg} / \mathrm{dl})$ & $\geq 50$ ys & 24 & 3.50 & 73.97 & $1.10(1.46)$ & 200 \\
\hline & $<50$ ys & 26 & -28.03 & 68.38 & $=0.30$ & 0.02 \\
\hline
\end{tabular}




\section{Continued}

\begin{tabular}{|c|c|c|c|c|c|c|}
\hline \multirow{4}{*}{$\begin{array}{l}\text { Creatinine } \\
(\mathrm{mg} / \mathrm{dl})\end{array}$} & Females & 31 & -0.02 & 0.15 & $0.16(1.46)$ & \multirow{2}{*}{0.003} \\
\hline & Males & 19 & -0.03 & 0.11 & $=0.69$ & \\
\hline & $\geq 50$ ys & 24 & -0.04 & 0.11 & $0.96(1.46)$ & \multirow{2}{*}{0.02} \\
\hline & $<50 \mathrm{ys}$ & 26 & -0.02 & 0.15 & $=0.33$ & \\
\hline \multirow{4}{*}{$\begin{array}{l}\text { Albumin } \\
\text { (gdl) }\end{array}$} & Females & 31 & -0.07 & 0.55 & $0.04(1.46)$ & \multirow{2}{*}{0.001} \\
\hline & Males & 19 & -0.05 & 0.54 & $=0.84$ & \\
\hline & $\geq 50$ ys & 24 & -0.05 & 0.53 & $0.11(1.46)$ & \multirow{2}{*}{0.003} \\
\hline & $<50$ ys & 26 & -0.07 & 0.57 & $=0.73$ & \\
\hline \multirow{4}{*}{$\begin{array}{c}\text { C Reactive } \\
\text { Protein } \\
(\mathrm{mg} / \mathrm{L})\end{array}$} & Females & 31 & -0.56 & 2.44 & $0.03(1.46)$ & \multirow{2}{*}{0.001} \\
\hline & Males & 19 & -0.35 & 2.33 & $=0.85$ & \\
\hline & $\geq 50 \mathrm{ys}$ & 24 & -0.42 & 2.35 & $0.03(1.46)$ & \multirow{2}{*}{0.001} \\
\hline & $<50 \mathrm{ys}$ & 26 & -0.54 & 2.45 & $=0.84$ & \\
\hline \multirow{4}{*}{$\begin{array}{l}\text { Fibrinogen } \\
\text { (mg/dl) }\end{array}$} & Females & 31 & 32.58 & 107.45 & 5.38 (1.46), & \multirow{2}{*}{0.10} \\
\hline & Males & 19 & -30.89 & 79.57 & $=0.02^{*}$ & \\
\hline & $\geq 50$ ys & 24 & -5.62 & 116.87 & \multirow{2}{*}{$\begin{array}{l}1.42(1.46) \\
=0.23\end{array}$} & \multirow{2}{*}{0.03} \\
\hline & $<50 \mathrm{ys}$ & 26 & 21.47 & 85.91 & & \\
\hline \multirow{4}{*}{$\begin{array}{c}\text { Glycated } \\
\text { Hemoglobin } \\
\text { (mgdl) }\end{array}$} & Females & 31 & -0.24 & 0.71 & $1.72(1.46)$ & \multirow{2}{*}{0.03} \\
\hline & Males & 19 & 0.01 & 0.76 & $=0.19$ & \\
\hline & $\geq 50$ ys & 24 & -0.05 & 0.73 & $1.09(1.46)$ & \\
\hline & $<50$ ys & 26 & -0.22 & 0.74 & $=0.30$ & 0.02 \\
\hline \multirow{4}{*}{$\begin{array}{l}\text { Salivary Cortisol } \\
\text { (nM) }\end{array}$} & Females & 31 & -3.82 & 5.31 & $0.01(1.46)$ & \multirow{2}{*}{0.001} \\
\hline & Males & 19 & -3.43 & 11.19 & $=0.90$ & \\
\hline & $\geq 50$ ys & 24 & -2.34 & 9.26 & 0.48(1.46), & 0.01 \\
\hline & $<50$ ys & 26 & -4.90 & 6.48 & $=0.49$ & 0.01 \\
\hline \multirow{4}{*}{$\begin{array}{l}\text { Salivary MHPG } \\
\text { (nM) }\end{array}$} & Females & 31 & -1103.93 & 1455.89 & $0.36(1.46)$ & \multirow{2}{*}{0.006} \\
\hline & Males & 19 & -1506.05 & 2646.08 & $=0.54$ & \\
\hline & $\geq 50$ ys & 24 & -1104.54 & 1511.31 & 0.27 (1.46), & 0.006 \\
\hline & $<50$ ys & 26 & -1397.23 & 2348.81 & $=0.60$ & \\
\hline \multirow{4}{*}{$\begin{array}{l}\text { Serum DHEA } \\
(\mathrm{ng} / \mathrm{ml})\end{array}$} & Females & 31 & -10.35 & 25.14 & 3.34 (1.46), & \multirow{2}{*}{0.06} \\
\hline & Males & 19 & 2.27 & 15.78 & $=0.07$ & \\
\hline & $\geq 50$ ys & 24 & -8.71 & 26.15 & 0.65 (1.46), & 0.01 \\
\hline & $<50$ ys & 26 & -2.63 & 19.11 & $=0.042^{*}$ & \\
\hline \multirow{4}{*}{$\begin{array}{l}\text { Serum NA } \\
\quad(\mathrm{pg} / \mathrm{ml})\end{array}$} & Females & 31 & -22.20 & 158.95 & 0.773 (1.46), & \multirow{2}{*}{0.017} \\
\hline & Males & 19 & -52.83 & 113.66 & $=0.38$ & \\
\hline & $\geq 50$ ys & 24 & -29.88 & 134.47 & 0.110(1.46), & \multirow{2}{*}{0.002} \\
\hline & $<50$ ys & 26 & -37.49 & 152.96 & $=0.74$ & \\
\hline
\end{tabular}

$\mathrm{n}$ : number of subjects per group; $\chi$ : average value; SD: standard deviation from mean; df: degrees of freedom, basal: week 0 of treatment, w12: week 12 of treatment, ys: years, MHPG: Methoxy-hydroxy-phenylglycol, DHEA: dehidro-epiandrosterone, NA: noradrenaline, ${ }^{*} \mathrm{p}<0.05$ (significant outcome). 
patients with GAD, are consistent with the literature review comparing sex effect on metabolic syndrome and/or on cardiovascular risk in general populations [7] [33]. However, there are only a few studies applying the AL index in patients with psychiatric disorders [19] [21].

As well as younger patients ( $<50$ years) but non-sex related factor, were significantly associated with lower total AL index after treatment, in this study many differences were found regarding sex. Women showed higher anxiety levels before and after treatment. It has been postulated that women may be more vulnerable and/or more exposed to chronic stress, to explain the higher incidence and severity of stress-related psychiatric disorders such as depression and/or anxiety [25] [34]. It has been well established that anxiety, depression and posttraumatic stress disorders, are highly influenced by sex and gonadal hormones [26] [35]. Regarding anxiety disorders, panic disorder is more common in women than in men, and females demonstrated a greater stress response [36]. Moreover, posttraumatic stress disorders are more frequently diagnosed among women [37]. In this study we found that anxiety levels were higher among women, suggesting a more severe psychiatric symptomatology. Nevertheless, women also showed a higher score in kindness in the neuroticism scale, which may constitute a more protective trait of personality.

Oppositely to psychiatric aspects, women showed a better AL profile among some individual allostatic variables, including cardiovascular, metabolic and obesity risk variables. These findings are consistent with other studies that compared sex differences analyzing cardiovascular risk factors and/or metabolic syndrome in general populations [19] [20] [36]. Males disadvantages for AL related pathologies, specially cardiovascular diseases, have been frequently observed across human populations; however, it is also well known that the sex gap is more pronounced in young adults and decreases in the postmenopausal stage coinciding with female fecundity decline [19] [20] [35]. Regarding this finding, in this study we grouped patients according to age with the cut off at 50 years to differentiate menopause factor. Nonetheless, there are some studies that showed higher inflammatory markers such as CRP levels with an inflammatory over response among women [19] [20] [38]. Similarly, in this study, women showed a better profile before and after treatment in cardiovascular and obesity factors but treatment seemed to bring a greater benefit to men by reducing both cholesterol ratio and fibrinogen, suggesting differences in treatment response. Furthermore, in women but not in men, a negative correlation between CRP levels with plasmatic cortisol levels after treatment was reported, suggesting a possible difference in the anti-inflammatory actions of cortisol regarding sex.

In this analysis, no differences between sex and age were observed regarding MHPG, (the principal noradrenergic metabolite and a biological marker of anxiety and chronic stress). MHPG was significantly reduced after treatment in all grouped patients with GAD. Similarly to our results, other studies reported variations in MHPG in patients with anxiety disorders [9] [39] [40]. In this study, we found a progressive reduction in salivary MHPG levels during 12 weeks of 
treatment without tolerance to this effect. Other researchers determined lower salivary levels of MHPG in anxious patients treated with alprazolam [9] [39] [40] and in normal subjects who received the drug [41]. Additionally experimental data has suggested that the response of CNS (Central Nervous System) amplifies the emotional response, and anxiety can be particularly regarded as an undifferentiated form of fear or rage discharged by noradrenaline [42].

This study showed blood pressure decay that returned to more normal levels with an additional reduction of anxiety and MHPG levels. Anxiety by itself has been proposed as an important factor involved in blood pressure elevation mediated by catecholamines and benzodiazepines, and particularly alprazolam has demonstrated anti-hypertensive pleiotropic properties especially in acute intervention and in patients with high blood pressure without affecting the heart rate [43] [44]. In relation to this finding a lower blood pressure was detected (systolic and diastolic in women, and only systolic in men), following 12 weeks of alprazolam treatment. It has been suggested that alprazolam in contrast with other anxiolytics as lorazepam has an additional suppression effect on the adrenomedullary system reducing the sympathetic discharge with lower plasma catecholamine's concentrations during exercise, stress, and throughout mental load (performing a cognitive test) [44] [45]. The high-potency benzodiazepine alprazolam (a triazolo-benzodiazepine), leads to a positive allosteric modulation of GABA-A receptor with an additional indirect activity through 5-HT1A serotoninergic receptors. These mechanisms are involved in the regulation of the hyperactive NA pathways inducing a reduction of noradrenergic system. It has been proposed that alprazolam administered at low doses may cause less impact on cognitive functions due to the shorter action compared to other benzodiazepines [46] [47]. Notwithstanding, the risk of inducing pharmacological dependence and/or other cognitive adverse events should be considered with a subsequent continuous monitoring [46] [48]. On the other side, chronic stress impairs cognitive function and decreases the likelihood of a prompt response to psychotherapy with the ensuing negative impact on quality of life [6].

Limitations of this study must be mentioned: Neither placebo nor untreated groups were included; since it is not acceptable for phase IV trials to include this type of highly symptomatic patients, and hence, it was necessary to administer the active drug to the entire sample and according to demand. The fact is passable for being a preliminary trial conducted to explore a concept and the overall safety. Controlled studies will be performed in the coming steps and different goals. In this study we did not report the cognitive variables (described in the original protocol and still under analysis) [27], which are planned for future evaluation. Furthermore, as a significant reduction in the total AL index was observed and many AL variables were significantly reduced after treatment, in this open study other non-pharmacological factors may also be considered to be interacting during treatment. Therefore, we cannot attribute the observations to a single drug effect. 


\section{Conclusion}

In this preliminary study we described sex and age differences in clinical psychiatric variables and parameters of AL index measured in patients with general anxiety disorders treated with alprazolam. These observations were scarce in the literature and will allow us to continue studying the impact of sex and age differences during the use of drugs for more protracted periods.

\section{Acknowledgements}

This study was funded with a partial grant from Gador SA, Buenos Aires (2012-2014 Grant) and partial funds of the Henri Laborit Institute of Biosciences, Córdoba, Argentina. We thank Pablo Lombardo for his helpful discussion related to cardiovascular and metabolic variables. Partial data was submitted at the European Congress of Neuropsychopharmacology 2017, 2018.

\section{Contributors}

All the co-authors have made substantial contributions to conception and design. LD has been involved in drafting and revising the manuscript for intellectual content. EJAR has given final approval of the version to be published.

\section{Funding and Declaration of Conflicting Interests}

A grant from Gador SA supported this study. CS and LD integrated the grant and EJAR is an investigator employed by Gador SA. CR does not declare any conflict of interest.

\section{Supplementary Materials}

Supplementary materials are available at Scientific Direction Department, Gador SA, Darwin 423, Buenos Aires, Argentina

\section{References}

[1] Crimmins, E.M., Johnston, M., Hayward, M. and Seeman, T. (2003) Age Differences in Allostatic Load: An Index of Physiological Dysregulation. Experimental Gerontology, 38, 731-734. https://doi.org/10.1016/S0531-5565(03)00099-8

[2] McEwen, B.S. (2005) Stressed or Stressed out: What Is the Difference? Journal of Psychiatry \& Neuroscience, 30, 315-318.

[3] McEwen, B.S. (2017) Neurobiological and Systemic Effects of Chronic Stress. Chronic Stress, 1, 1-17. https://doi.org/10.1177/2470547017692328

[4] Danese, A. and McEwen, B.S. (2012) Adverse Childhood Experiences, Allostasis, Allostatic Load, and Age-Related Disease. Physiology \& Behavior, 106, 29-39. https://doi.org/10.1016/j.physbeh.2011.08.019

[5] McEwen, B.S. (2003) Mood Disorders and Allostatic Load. Biological Psychiatry, 54, 200-207. https://doi.org/10.1016/S0006-3223(03)00177-X

[6] Karatsoreos, I.N. and McEwen, B.S. (2013) Resilience and Vulnerability: A Neurobiological Perspective. F1000Prime Reports, 5, 13.

[7] Seeman, T., Epel, E., Gruenewald, T., Karlamangla, A. and McEwen, B.S. (2010) So- 
cio-Economic Differentials in Peripheral Biology: Cumulative Allostatic Load. Annals of the New York Academy of Sciences, 1186, 223-239. https://doi.org/10.1111/j.1749-6632.2009.05341.x

[8] Chaudieu, I., Beluche, I., Norton, J., Boulenger, J.-P., Ritchie, K. and Ancelin, M.L. (2008) Abnormal Reactions to Environmental Stress in Elderly Persons with Anxiety Disorders: Evidence from a Population Study of Diurnal Cortisol Changes. Jour nal of Affective Disorders, 106, 307-313. https://doi.org/10.1016/j.jad.2007.07.025

[9] Curtis, G.C., Abelson, J.L. and Gold, P.W. (1997) Adrenocorticotropic Hormone and Cortisol Responses to Corticotropin-Releasing Hormone: Changes in Panic Disorder and Effects of Alprazolam Treatment. Biological Psychiatry, 41, 76-85. https://doi.org/10.1016/S0006-3223(95)00578-1

[10] de Kloet, E.R., Otte, C., Kumsta, R., Kok, L., Hillegers, M.H.J., Hasselmann, H., Kliegel, D. and Joëls, M. (2016) Stress and Depression a Crucial Role of the Mineralocorticoid Receptor. Journal of Neuroendocrinology, 28, 1-12. https://doi.org/10.1111/jne.12379

[11] Rüther, E., Wedekind, D., Bandelow, B., Broocks, A. and Hajak, G. (2000) Salivary, Total Plasma and Plasma Free Cortisol in Panic Disorder. Journal of Neural Trans mission, 107, 831-817. https://doi.org/10.1007/s007020070062

[12] Mantella, R.C., Butters, M.A., Amico, J.A., Mazumdar, S., Rollman, B.L., Begley, A.E., Reynolds, C.F. and Lenze, E.J. (2008) Salivary Cortisol Is Associated with Diagnosis and Severity of Late-Life Generalized Anxiety Disorder. Psychoneuroendocrinology, 33, 773-781. https://doi.org/10.1016/j.psyneuen.2008.03.002

[13] Garcia-Banda, G., Chellew, K., Fornes, J., Perez, G., Servera, M. and Evans, P. (2014) Neuroticism and Cortisol: Pinning down an Expected Effect. International Journal of Psychophysiology, 91, 132-138. https://doi.org/10.1016/j.ijpsycho.2013.12.005

[14] de Kloet, E.R., Joëls, M. and Holsboer, F. (2005) Stress and the Brain: From Adaptation to Disease. Nature Reviews Neuroscience, 6, 463-475. https://doi.org/10.1038/nrn1683

[15] McEwen, B.S. and Gianaros, P.J. (2011) Stress-and Allostasis-Induced Brain Plasticity. Annual Review of Medicine, 62, 431-445. https://doi.org/10.1146/annurev-med-052209-100430

[16] Hoge, E.A., Ivkovic, A. and Fricchione, G.L. (2012) Generalized Anxiety Disorder: Diagnosis and Treatment. BMJ, 345, e7500. https://doi.org/10.1136/bmj.e7500

[17] Verma, C.S., Balhara, R. and Gupta, Y.P.S. (2011) Gender Differences in Stress Response: Role of Developmental and Biological Determinants. Industrial Psychiatry Journal, 20, 4-10.

[18] Fezeu, J.C., Balkau, L., Kengne, B., Sobngwi, A.P. and Mbanya, E. (2007) Metabolic Syndrome in a Sub-Saharan African Setting: Central Obesity May Be the Key Determinant. Atherosclerosis, 193, 70-76. https://doi.org/10.1016/j.atherosclerosis.2006.08.037

[19] Yang, Y. and Kozloski, M. (2011) Dysregulation: Inflammation, Metabolic Syndrome, and Allostatic Load. Journals of Gerontology, 66, 493-500.

[20] Ahonen, T., Saltevo, J., Laakso, M., Kautiainen, H., Kumpusalo, E. and Vanhala, M. (2009) Gender Differences Relating to Metabolic Syndrome and Proinflammation in Finnish Subjects with Elevated Blood Pressure. Mediators of Inflammation, 2009, Article ID: 959281.

[21] Juster, R.-P., Pruessner, J.C., Desrochers, A.B., Bourdon, O., Durand, N., Wan, N., Tourjman, V., Kouassi, E., Lesage, A. and Lupien, S.J. (2016) Sex and Gender Roles 
in Relation to Mental Health and Allostatic Load. Psychosomatic Medicine, 78, 788-804. https://doi.org/10.1097/PSY.0000000000000351

[22] Seeman, T.E., Crimmins, E., Huang, M.H., Singer, B., Bucur, A., Gruenewald, T., Berkman, L.F. and Reuben, D.B. (2004) Cumulative Biological Risk and Socio-Economic Differences in Mortality: MacArthur Studies of Successful Aging. Social Science \& Medicine, 58, 1985-1997. https://doi.org/10.1016/S0277-9536(03)00402-7

[23] Manji, B.H.K. and Duman, R.S. (2001) Impairments of Neuroplasticity and Cellular Resilience in Severe Mood Disorders: Implications for the Development of Novel Therapeutics. Spring, 35, 5-49.

[24] Heaney, J.L.J., Phillips, A.C. and Carroll, D. (2010) Ageing, Depression, Anxiety, Social Support and the Diurnal Rhythm and Awakening Response of Salivary Cortisol. International Journal of Psychophysiology, 78, 201-208. https://doi.org/10.1016/j.ijpsycho.2010.07.009

[25] Hek, K., Direk, N., Newson, R.S., Hofman, A., Hoogendijk, W.J.G., Mulder, C.L. and Tiemeier, H. (2013) Anxiety Disorders and Salivary Cortisol Levels in Older Adults: A Population-Based Study. Psychoneuroendocrinology, 38, 300-305. https://doi.org/10.1016/j.psyneuen.2012.06.006

[26] Juster, R., Mcewen, B.S. and Lupien, S.J. (2010) Neuroscience and Biobehavioral Reviews Allostatic Load Biomarkers of Chronic Stress and Impact on Health and Cognition. Neuroscience \& Biobehavioral Reviews, 35, 2-16.

https://doi.org/10.1016/j.neubiorev.2009.10.002

[27] Soria, C.A., Remedi, C., Núñez, D., D’Alessio, L. and Roldán, E.J. (2015) Impact of Alprazolam in Allostatic Load and Neurocognition of Patients with Anxiety Disorders and Chronic Stress (GEMA): Observational Study Protocol. BMJ Open, 5, e007231. https://doi.org/10.1136/bmjopen-2014-007231

[28] Hamilton, M. (1969) Diagnosis and Ratings of Anxiety. British Journal of Psychiatry, 3, 76-79.

[29] Costa, R.R. and McCrae, P.T. (1999) Inventario de Personalidad NEO Revisado (NEO PI-R), Inventario NEO Reducido de Cinco Factores (NEO-FFI). Man. Prof.

[30] Folstein, M.F. (1975) "Mini-Mental State": A Practical Method for Grading the Mental State of Patients for the Clinician. Journal of Psychiatric Research, 12, 189-198. https://doi.org/10.1016/0022-3956(75)90026-6

[31] American Psychiatric Asocciation (1994) Diagnostic and Statistical Manual of Mental Disorders. DSM IV. 4th Edition, American Psychiatric Association, Washington DC.

[32] Granato, D., de Araújo Calado, V.Ô.M. and Jarvis, B. (2014) Observations on the Use of Statistical Methods in Food Science and Technology. Food Research International, 55, 137-149. https://doi.org/10.1016/j.foodres.2013.10.024

[33] Malik, S., Wong, N.D., Franklin, S.S., Kamath, T.V., Italien, G.J.L., Pio, J.R. and Williams, G.R. (2004) Coronary Heart Disease, Cardiovascular Disease, and All Causes in United States Adults. Circulation, 110, 1245-1251. https://doi.org/10.1161/01.CIR.0000140677.20606.0E

[34] Burke, D.C., Davis, H.M., Otte, M.C. and Mohr, C. (2005) Depression and Cortisol Responses to Psychological Stress: A Meta-Analysis. Psychoneuroendocrinology, 30, 846-856. https://doi.org/10.1016/j.psyneuen.2005.02.010

[35] Paris, J.J., Franco, C., Sodano, R., Freidenberg, B., Gordis, E., Anderson, D.A., Forsyth, J.P., Wulfert, E. and Frye, C.A. (2011) Sex Differences in Salivary Cortisol in Response to Acute Stressors among Healthy Participants, in Recreational or Patho- 
logical Gamblers, and in Those with Posttraumatic Stress Disorder. Hormones and Behavior, 57, 35-45.

[36] Hellhammer, B.M., Wüst, D.H. and Kudielka, S. (2009) Salivary Cortisol as a Biomarker in Stress Research. Psychoneuroendocrinology, 34, 163-171.

https://doi.org/10.1016/j.psyneuen.2008.10.026

[37] Aardal-Eriksson, L.H., Eriksson, E., and Thorell, T.E. (2001) Salivary Cortisol, Posttraumatic Stress Symptoms, and General Health in the Acute Phase and during 9-Month Follow-Up. Biological Psychiatry, 50, 986-993. https://doi.org/10.1016/S0006-3223(01)01253-7

[38] Lakoski, D.M., Cushman, S.G., Criqui, M., Rundek, M., Blumenthal, T., D’agostino, R.S. and Herrington, R.B. (2006) Gender and C-Reactive Protein: Data from the Multiethnic Study of Atherosclerosis (MESA) Cohort. American Heart Journal, 152, 593-598. https://doi.org/10.1016/j.ahj.2006.02.015

[39] Yamada, S., Yamauchi, K., Yajima, J., Hisadomi, S., Maeda, H., Toyomasu, K. and Tanaka, M. (2000) Saliva Level of Free 3-Methoxy-4-Hydroxyphenylglycol (MHPG) as a Biological Index of Anxiety Disorders. Psychiatry Research, 93, 217-223. https://doi.org/10.1016/S0165-1781(00)00118-9

[40] Fries, E., Hellhammer, D.H. and Hellhammer, J. (2006) Attenuation of the Hypothalamic-Pituitary-Adrenal Axis Responsivity to the Trier Social Stress Test by the Benzodiazepine Alprazolam. Psychoneuroendocrinology, 31, 1278-1288. https://doi.org/10.1016/j.psyneuen.2006.09.009

[41] Davidson, M., Zemishlany, Z., McQueeney, R. and Gabriel, S.M. (1990) Neuroendocrine and Monoaminergic Responses to Acute Administration of Alprazolam in Normal Subjects. Neuropsychobiology, 23, 124-128. https://doi.org/10.1159/000119437

[42] Yamamoto, K.I., Shinba, T. and Yoshii, M. (2014) Psychiatric Symptoms of Noradrenergic Dysfunction: A Pathophysiological View. Psychiatry and Clinical Neurosciences, 68, 1-20. https://doi.org/10.1111/pcn.12126

[43] Yilmaz, S., Pekdemir, M., Tural, U. and Uygun, M. (2011) Comparison of Alprazolam versus Captopril in High Blood Pressure: A Randomized Controlled Trial. Blood Pressure, 20, 239-243. https://doi.org/10.3109/08037051.2011.553934

[44] Van Den Berg, F., Tulen, J.H.M., Boomsma, F., Noten, J.B.G.M., Moleman, P. and Pepplinkhuizen, L. (1996) Effects of Alprazolam and Lorazepam on Catecholaminergic and Cardiovascular Activity during Supine Rest, Mental Load and Orthostatic Challenge. Psychopharmacology, 128, 21-30. https://doi.org/10.1007/s002130050105

[45] Stratton J.R. and Halter, J.B. (1985) Effect of a Benzodiazepine (Alprazolam) on Plasma Epinephrine and Norepinephrine Levels during Exercise Stress. American Journal of Cardiology, 56, 136-139. https://doi.org/10.1016/0002-9149(85)90582-X

[46] Billioti, S., Gage, D., Bégaud, B. and Bazin, F. (2012) Benzodiazepine Use and Risk of Dementia: Prospective Population Based Study. BMJ, 345, e6231-e6231.

[47] Lüscher, C., Tan, K.R. and Rudolph, U. (2011) Hooke on Benzodiazepines: GABAA Receptor Subtypes and Addiction. Trends in Neurosciences, 34, 188-197. https://doi.org/10.1016/j.tins.2011.01.004

[48] Barker, M.J., Greenwood, K.M., Jackson, M. and Crowe, S.F. (2004) Persistence of Cognitive Effects after Withdrawal from Long-Term Benzodiazepine Use: A Meta-Analysis. Archives of Clinical Neuropsychology, 19, 437-454. https://doi.org/10.1016/S0887-6177(03)00096-9 